\title{
Improvement of the Nitration Process in 2-Amino-5-chloro-4-methylbenzenesulfonic Acid Synthesis
}

Hai-Ming Li, Guang Han, Shi-Liang Liu, Yuan-Xiao Li, Feng-Bao Zhang, Guo-Liang Zhang, Qing Xia*

School of Chemical Engineering and Technology, Tianjin University, Tianjin 300072, PR China

\section{Supporting Information}

Table S1. Molality solubility data of CNTNa $\left(m_{1}\right)$ in different solute-free mass fraction of sulfuric acid $\left(w_{2}^{0}\right)$ for the system of $\{\mathrm{CNTNa}(1)+$ sulfuric acid $(2)+$ water (3) $\}$ at temperature $T^{\exp }$ and pressure $0.1 \mathrm{MPa}$.

Figure S1. XRD pattern of CNTNa: A representative of after dissolution; B representative of before dissolution.

* Corresponding author.

Tel.: +86-22-27400292; Fax: +86-22-27408778.

E-mail address: xiaqing@tju.edu.cn. (Qing XIA) 


\section{Improvement of the Nitration Process in}

\section{2-Amino-5-chloro-4-methylbenzenesulfonic Acid Synthesis}

Hai-Ming Li, Guang Han, Shi-Liang Liu, Yuan-Xiao Li, Feng-Bao Zhang, Guo-Liang Zhang, Qing Xia*

School of Chemical Engineering and Technology, Tianjin University, Tianjin 300072, PR China

Table S1. Molality solubility data of CNTNa $\left(m_{1}\right)$ in different solute-free mass fraction of sulfuric acid $\left(w_{2}^{0}\right)$ for the system of $\{\mathrm{CNTNa}(1)+$ sulfuric acid $(2)+$ water (3) $\}$ at temperature $T^{\exp }$ and pressure $0.1 \mathrm{MPa}$.

\begin{tabular}{|c|c|c|c|c|c|}
\hline$m_{1} /\left(\mathrm{mol} \cdot \mathrm{kg}^{-1}\right)$ & $T^{\exp } / \mathrm{K}$ & $m_{1} /\left(\mathrm{mol} \cdot \mathrm{kg}^{-1}\right)$ & $T^{\exp } / \mathrm{K}$ & $m_{1} /\left(\mathrm{mol} \cdot \mathrm{kg}^{-1}\right)$ & $T^{\exp } / \mathrm{K}$ \\
\hline \multicolumn{6}{|c|}{$w_{2}^{0}=0.00$} \\
\hline 0.1169 & 278.55 & 0.4376 & 304.47 & 1.412 & 327.53 \\
\hline 0.1494 & 283.86 & 0.5598 & 309.34 & 1.830 & 332.79 \\
\hline 0.1786 & 288.97 & 0.7021 & 313.43 & 2.385 & 337.82 \\
\hline 0.2337 & 293.60 & 0.8839 & 318.29 & 3.118 & 342.34 \\
\hline 0.3326 & 299.56 & 1.094 & 322.31 & & \\
\hline \multicolumn{6}{|c|}{$w_{2}^{0}=0.20$} \\
\hline $9.921 \cdot 10^{-3}$ & 277.53 & 0.2368 & 302.76 & 1.036 & 329.16 \\
\hline $1.643 \cdot 10^{-2}$ & 281.56 & 0.3011 & 306.62 & 1.297 & 333.91 \\
\hline $3.167 \cdot 10^{-2}$ & 285.67 & 0.4082 & 310.81 & 1.578 & 338.65 \\
\hline $6.361 \cdot 10^{-2}$ & 290.05 & 0.5114 & 315.52 & 1.895 & 343.48 \\
\hline 0.1097 & 293.68 & 0.6814 & 320.77 & & \\
\hline 0.1581 & 298.34 & 0.8748 & 325.43 & & \\
\hline \multicolumn{6}{|c|}{$w_{2}^{0}=0.40$} \\
\hline $0.5025 \cdot 10^{-3}$ & 277.58 & $5.779 \cdot 10^{-3}$ & 301.28 & $6.897 \cdot 10^{-2}$ & 322.02 \\
\hline $1.048 \cdot 10^{-3}$ & 282.74 & $8.907 \cdot 10^{-3}$ & 305.49 & 0.1089 & 326.19 \\
\hline $1.716 \cdot 10^{-3}$ & 287.74 & $1.383 \cdot 10^{-2}$ & 309.94 & 0.1531 & 329.24 \\
\hline $2.908 \cdot 10^{-3}$ & 292.41 & $2.225 \cdot 10^{-2}$ & 313.90 & 0.3514 & 337.85 \\
\hline $4.399 \cdot 10^{-3}$ & 296.89 & $3.843 \cdot 10^{-2}$ & 317.01 & 0.5844 & 342.49 \\
\hline \multicolumn{6}{|c|}{$w_{2}^{0}=0.60$} \\
\hline $0.4574 \cdot 10^{-3}$ & 278.86 & $5.127 \cdot 10^{-3}$ & 305.25 & $2.048 \cdot 10^{-2}$ & 330.14 \\
\hline $0.8442 \cdot 10^{-3}$ & 282.95 & $6.691 \cdot 10^{-3}$ & 309.83 & $2.593 \cdot 10^{-2}$ & 334.26 \\
\hline $1.431 \cdot 10^{-3}$ & 288.17 & $8.511 \cdot 10^{-3}$ & 314.39 & $3.506 \cdot 10^{-2}$ & 338.49 \\
\hline
\end{tabular}




\begin{tabular}{lccccc}
\hline $2.122 \cdot 10^{-3}$ & 292.94 & $1.056 \cdot 10^{-2}$ & 318.02 & $4.721 \cdot 10^{-2}$ & 342.81 \\
$2.908 \cdot 10^{-3}$ & 297.28 & $1.301 \cdot 10^{-2}$ & 321.86 & & \\
$3.972 \cdot 10^{-3}$ & 301.46 & $1.629 \cdot 10^{-2}$ & 326.22 & & \\
& \multicolumn{5}{c}{$w_{2}^{0}=0.70$} \\
$0.3819 \cdot 10^{-3}$ & 278.74 & $3.421 \cdot 10^{-3}$ & 304.96 & $1.139 \cdot 10^{-2}$ & 329.36 \\
$0.6699 \cdot 10^{-3}$ & 283.46 & $4.187 \cdot 10^{-3}$ & 309.04 & $1.436 \cdot 10^{-2}$ & 332.83 \\
$0.9837 \cdot 10^{-3}$ & 287.55 & $5.013 \cdot 10^{-3}$ & 313.12 & $1.912 \cdot 10^{-2}$ & 337.32 \\
$1.295 \cdot 10^{-3}$ & 291.77 & $6.158 \cdot 10^{-3}$ & 316.97 & $2.474 \cdot 10^{-2}$ & 342.07 \\
$1.770 \cdot 10^{-3}$ & 295.59 & $7.610 \cdot 10^{-3}$ & 321.18 & & \\
$2.344 \cdot 10^{-3}$ & 300.26 & $9.218 \cdot 10^{-3}$ & 324.93 & & \\
& & & & & \\
$0.8153 \cdot 10^{-3}$ & 278.63 & $1.707 \cdot 10^{-2}$ & 301.62 & 0.1468 & 322.51 \\
$2.057 \cdot 10^{-3}$ & 283.89 & $2.626 \cdot 10^{-2}$ & 305.98 & 0.2053 & 326.50 \\
$3.468 \cdot 10^{-3}$ & 288.33 & $4.528 \cdot 10^{-2}$ & 310.45 & 0.3209 & 331.77 \\
$6.385 \cdot 10^{-3}$ & 292.84 & $6.721 \cdot 10^{-2}$ & 313.91 & 0.5841 & 339.01 \\
$9.147 \cdot 10^{-3}$ & 297.22 & 0.1043 & 318.25 & 0.8692 & 343.27 \\
\hline
\end{tabular}

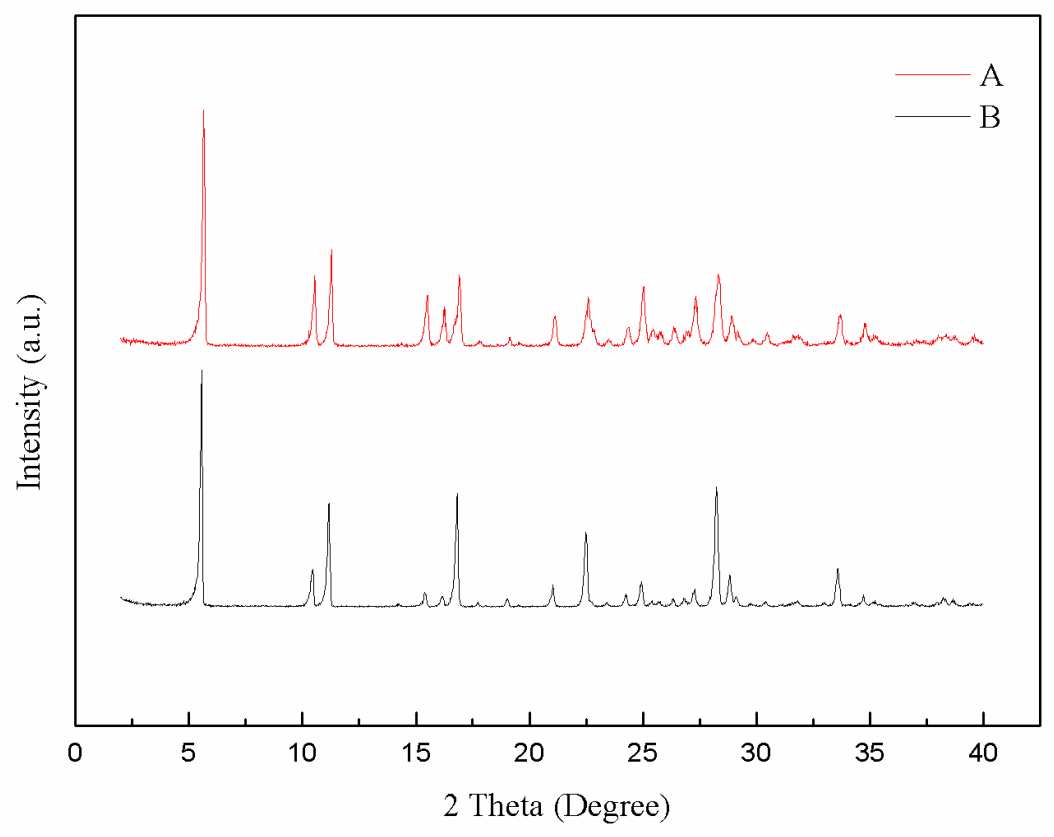

Figure S1. XRD pattern of CNTNa: A representative of after dissolution; B representative of before dissolution. 